\title{
Left bundle branch block and myocardial infarction, a diagnosis not always easy: Our experience and review of literature
}

\author{
Angela Sansone, Francesca Bonura, Fabiana Castellano, Rosanna Iacona, Dario Mancuso, \\ Giuseppina Novo, Pasquale Assennato, Salvatore Novo
}

Department of Internal Medicine, Cardiovascular and Nephro-Urological Disease, Unit of Cardiology, University of Studies "P. Giaccone" Palermo, Palermo, Italy

Email: angela.sansone84@yahoo.it

Received 11 January 2012; revised 8 March 2012; accepted 18 March 2012

\begin{abstract}
The occurrence of left bundle branch block (LBBB) is quite common in clinical practice. The changes in cardiac repolarization, caused by this disorder of electric conduction, may mask the presence of an acute myocardial infarction (AMI), delaying the diagnostic-therapeutic iter, with an important impact on prognosis. We describe the case of a woman of 59 years with LBBB, came to our observation for a constrictive chest pain associated with dyspnea. The diagnostic workup for suspected acute coronary syndrome (ACS), initially conducted only on the analysis of the electrocardiogram (negative TnI at entry), showed the presence of coronary arteries free of stenosis. However, the diagnostic confirmation of AMI was completed after the rise of cardiac markers and the electrocardiographic changes. This case confirm the difficulty about the diagnosis of AMI in patients with LBBB and stresses, however, as the use of some criteria proposed in the literature [1-3] can guide to its identification, directing patient to an appropriate treatment.
\end{abstract}

Keywords: Left Bundle Branch Block (LBBB); Myocardial Infarction; Electrocardiogram

\section{INTRODUCTION}

The occurrence of a right bundle branch block or a left one, is quite common in routine electrocardiograms (ECGs). In particular, the LBBB is often evident in patients with cardiac injury and/or systemic hypertension. The changes in cardiac repolarization in this disorder of electric conduction, may also mask the classical electrocardiographic changes of -ST segment in patients with AMI. For years, therefore, several authors have focused their attention on the management of patients with suspected ACS and LBBB on ECG [4-6], in order to identify electrocardiographic criteria which could allow the diagnosis of AMI fastest, optimizing at the same time, the results of reperfusion therapy $[7,8]$.

\section{CASE REPORT}

Woman of 59 years, hypertensive for about twenty years, smoker, with a positive family history for cardiovascular disease (father died for ACS). Comes to our attention in September 2008 for constrictive chest pain of high intensity associated with dyspnea. OE: $3 / 6 \mathrm{~L}$ rough systolic murmur on aortic outbreak, abolition of the second tone, VM harsh. BP: 130/70 mmHg. ECG: SR at frequency of 75 bpm, LBBB (Figure 1).

The patient also reports home therapy with: telmisar$\tan 80 \mathrm{mg}$, amlodipine $10 \mathrm{mg}$, cardioaspirin, omeprazole. Be reported to the blood tests performed in the E. R.: glycaemia $135 \mathrm{mg} / \mathrm{dl}$, neutrophilic leukocytosis (WBC $10.7 \times 10^{3} \mu \mathrm{L}$, NEUT $\left.8.7 \times 10^{3} \mu \mathrm{L}\right)$, LDH $458 \mathrm{UI} / \mathrm{L}, \mathrm{CPK}$ $59 \mathrm{UI} / \mathrm{L}, \mathrm{TnI} 0.017 \mathrm{ng} / \mathrm{ml}$. Given the severity and the persistence of chest pain and the electrocardiographic finding of LBBB, at the time to interpret for the presence of signs that could suggest an AMI in progress, was administered i.v therapy (ASA, heparin 4000UI, omeprazole and antibiotic prophylaxis) and moved the patient to Hemodynamic Unit to perform coronary angiography in emergency, that showed the absence of angiographically significant stenosis.

The seriated blood test performed during hospitalization, showed progressive increase in cardiac markers (peak value: LDH $611 \mathrm{UI} / \mathrm{L}, \mathrm{CPK} 317 \mathrm{UI} / \mathrm{L}$, TnI 9.02 $\mathrm{ng} / \mathrm{ml}$ ). Also be noted, sequential electrocardiographic changes (maximum elevation of the J point in V3: $5 \mathrm{~mm}$, V4: $4 \mathrm{~mm}, \mathrm{~V} 5: 3 \mathrm{~mm}$.) regressed on the second day of hospitalization (Figures 2 and 3). During hospitalization, 


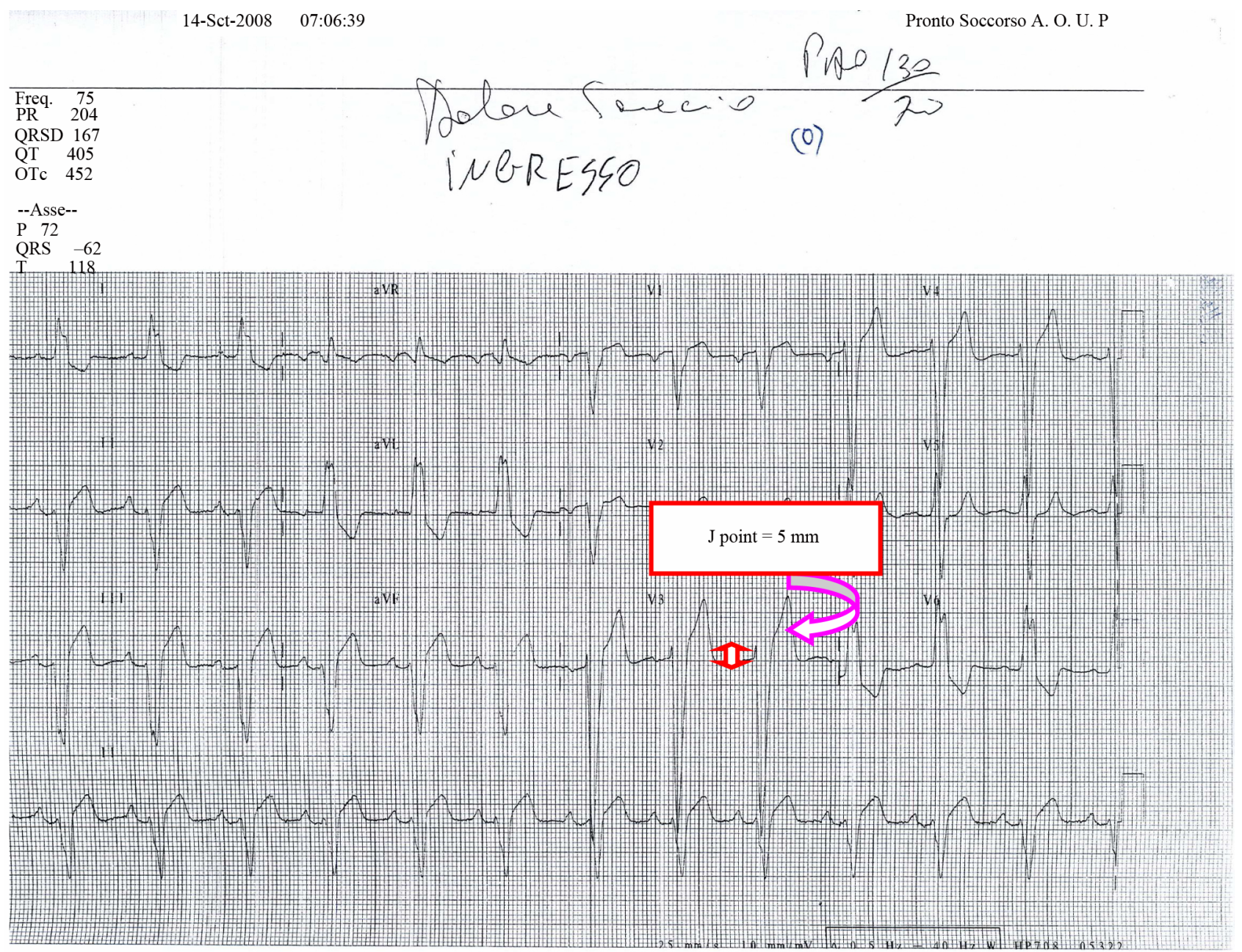

Figure 1. ECG at entry: J point elevation in $V 3$ of $5 \mathrm{~mm}$.

the patient also performs an echocardiogram of control that evidenced the presence of hypertensive cardiopathy, EF moderately depressed (42\%) and a moderate-severe aortic steno-insufficiency. During the remaining days of hospitalization, the patient did not reported new episodes of chest pain, with progressive normalization of electrocardiographic changes and cardiac markers (Figure 4).

The patient was discharged on fourth day with a diagnosis of AMI without ST-segment elevation and indication to surgical treatment of aortic valve disease.

\section{DISCUSSION}

The clinical case reported is suitable to some general considerations about the diagnosis of AMI in patients with complete LBBB. The recent evidence of a LBBB in a patient with chest pain, is in fact strongly suggestive of a heart attack, as well as electrocardiographic changes occurring on a chronic $\mathrm{LBBB}$ are easily recognizable when we have previous ECGs to compare. However, this observation doesn't always occur in clinical practice, therefore Sgarbossa et al. [9] proposed in 1996 electro- cardiographic criteria to diagnose an AMI in patients with chest pain and LBBB. In particular, the attention was focused on the deviation of the ST-segment. Each of the three criteria identified was also associated [10], through a multivariate analysis, with a value of Odd Ratio, that expresses the importance of each in predicting an AMI in progress, and with an index score ranging from 5 to 2 . The elevation of ST-segment $\geq 1 \mathrm{~mm}$ concordant with the QRS complex or the depression of the same $\geq 1 \mathrm{~mm}$ in leads V1, V2 or V3; are especially to be considered signs of an AMI, even without other electrocardiographic changes; while the elevation of ST-segment $\geq 5 \mathrm{~mm}$ discordant with the QRS complex, actually expresses only a moderate to high probability of AMI, therefore additional procedures are needed to confirm the diagnosis.

In the clinical case presented the diagnostic-therapeutic iter followed for the patient, it has been suggested by clinical conditions and on a first analysis of a electrocardiogram showing a J-point elevation in V3 of $5 \mathrm{~mm}$, that in absence of other criteria, corresponds to a probability 


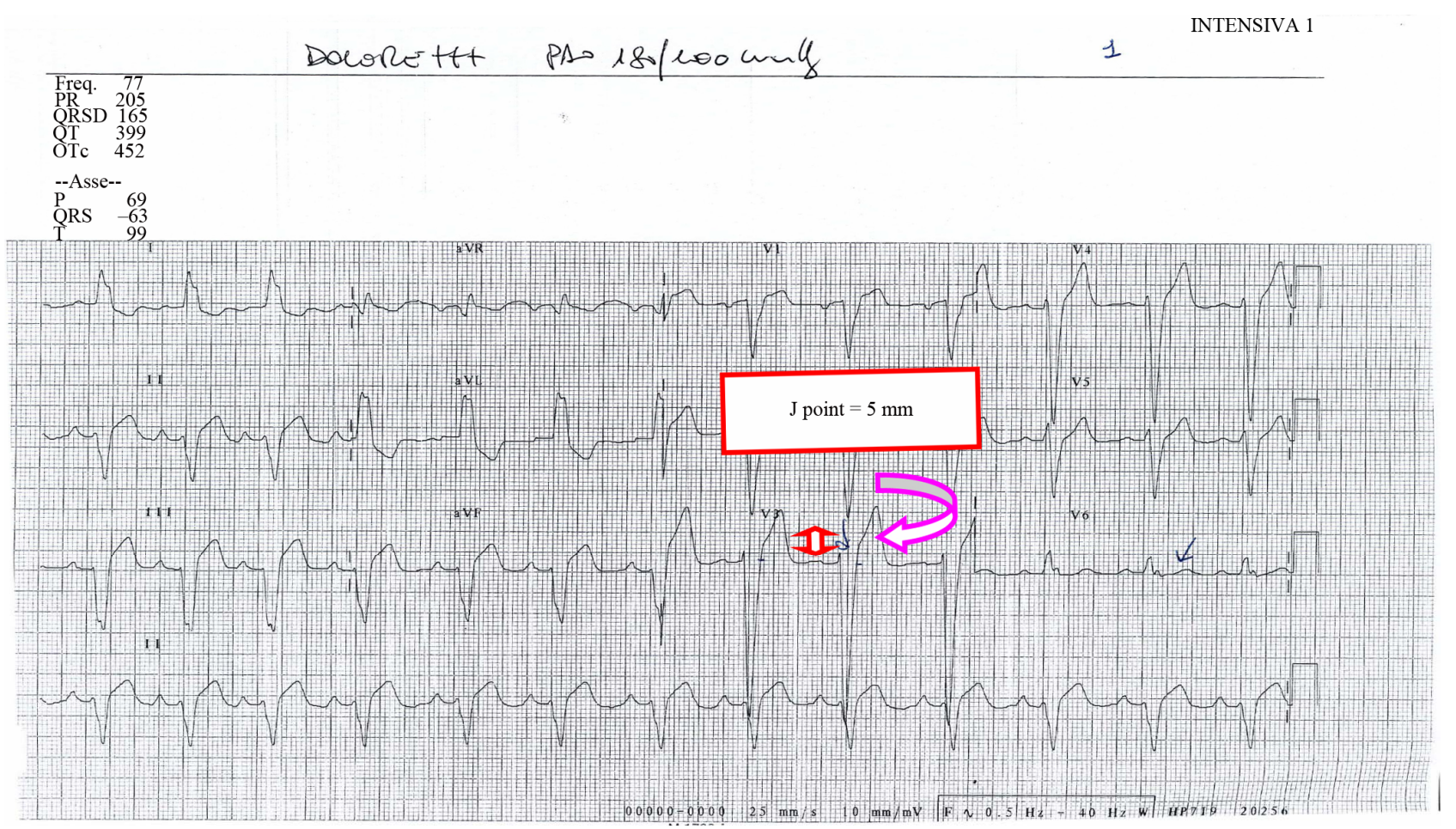

Figure 2. ECG: persistent $\mathrm{J}$ point elevation $\mathrm{J}=5 \mathrm{~mm}$.

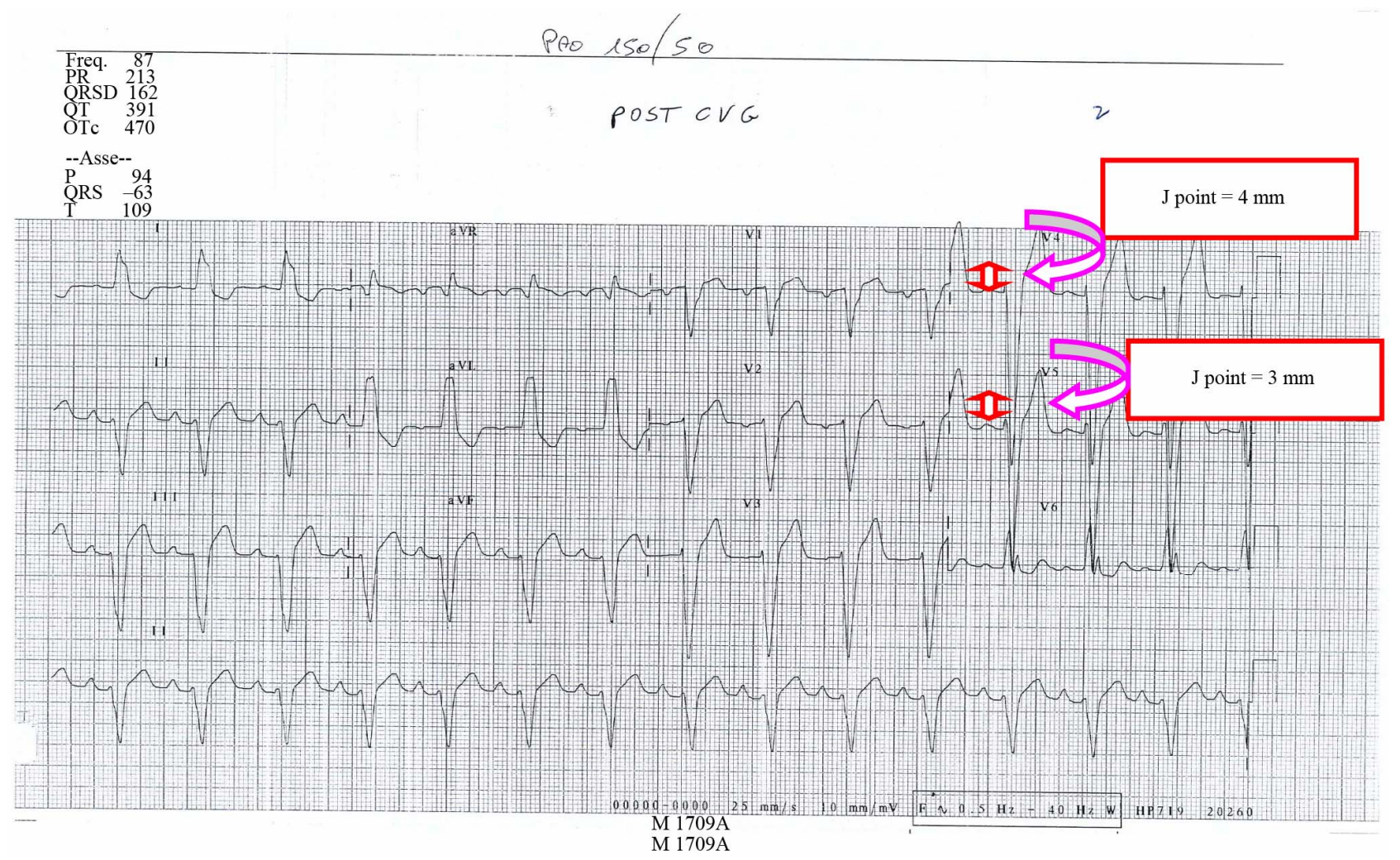

Figure 3. ECG: $\mathrm{J}$ point elevation in $\mathrm{V} 4=4 \mathrm{~mm}$ e in $\mathrm{V} 5=3 \mathrm{~mm}$.

of AMI by $50 \%$ and an index score of 2 [11]. The patient underwent therefore coronary angiography in emergency that showed the absence of angiographically significant stenosis, but the diagnostic confirmation of AMI was 


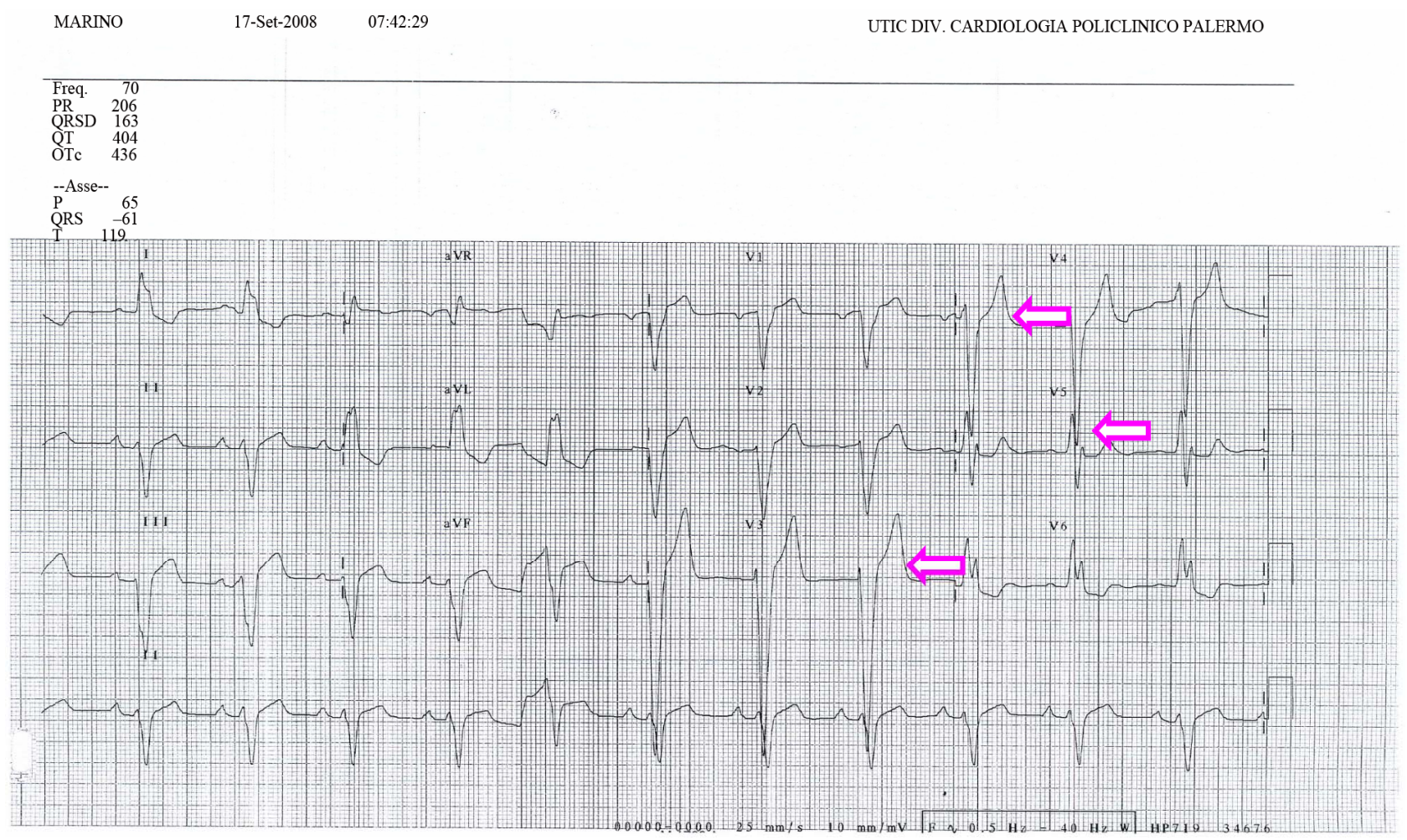

Figure 4. ECG at the discharge: regression of J point elevation from V3 to V5.

subsequently supported by electrocardiographic changes and the progressive rise in cardiac markers. The use of above criteria, helped therefore to identify a patient with AMI who could receive an adequate treatment.

The electrocardiographic criteria proposed by Sgarbossa were then revised in 2005 by Wong et al. that wanted to test impact of each criterion on the outcome of patients with LBBB and a new-onset chest pain, underwent thrombolytic therapy [12]. This analysis demonstrated a high specificity and low sensitivity for the first two criteria, reduced specificity and sensivity for the last one. In fact, although the criteria about the variation of ST-segment identified patients with AMI enzymatically confirmed, with higher levels of cardiac markers and worst 30-day mortality; the low sensitivity indicates that the absence of this criterion is not enough to rule out a enzymatically confirmation of AMI. Finally, patients didn't show ST-segment changes on the ECG had a better prognosis at 30 days.

Considering therefore, both of Sgarbossa's diagnostic flow chart and the Wong's prognostic analysis, it is deducted that the electrocardiographic analysis has certainly an important role in the diagnosis of AMI in patients with LBBB; this disorder also increases the risk of mortality in patients following an AMI and increasingly according to age and co-morbidities; so each of the criteria above discussed, has an impact on clinical outcome of patients following an AMI.

\section{CONCLUSION}

In patients with chest pain and LBBB, the finding of some electrocardiographic criteria may contribute significantly to the identification of acute myocardial infarction, especially in absence of previous medical documents and/or the support of the laboratory. The presence or no of the same ones, the timeliness of the treatment, also contribute, together with the common risk factors, to define the prognosis of patients following an AMI.

\section{REFERENCES}

[1] Gunnarsson, G., Eriksson, P. and Dellborg, M. (2001) ECG criteria in diagnosis of acute myocardial infarction in the presence of left bundle branch block. International Journal of Cardiology, 78, 167-174. doi:10.1016/S0167-5273(01)00378-3

[2] Kontos, M.C., McQueen, R.H., Jesse, R.L., et al. (2001) Can myocardial infarction be rapidly identified in emergency department patients who have left bundle-branch block? Annals of Emergency Medicine, 37, 431-438. doi: $10.1067 / \mathrm{mem} .2001 .114900$

[3] Antman, E.M., Anbe, D.T., Armstrong, P.W., et al. (2004) "ACC/AHA guidelines for the management of patients with ST-elevation myocardial infarction - executive summary and report of the ACC/AHA Task Force on Practice Guidelines (Writing Committee to Revise the 1999 Guidelines for the Management of Patients with Acute Myocardial Infarction). Circulation, 110, 588-636. 


$$
\text { doi:10.1161/01.CIR.0000134791.68010.FA }
$$

[4] Zimetbaum, P.J. and Josephson, M.E. (2003) Use of the electrocardiogram in acute myocardial infarction. New England Journal of Medicine, 348, 933-940. doi:10.1056/NEJMra022700

[5] Edhouse, J.A., Sakr, M., Angus, J., et al. (1999) Suspected myocardial infarction and left bundle branch block: Electrocardiographic indication of acute ischemia. Journal of Accident \& Emergency Medicine, 16, 331-335. doi:10.1136/emj.16.5.331

[6] Tabas, J.A., Rodriguez, R.M., Seligman, H.K. and Goldschlager, N.F. (2008) Electrocardiographic criteria for detecting acute myocardial infarction in patients with left bundle branch block: A meta-analysis. Annals of Emergency Medicine, 52, 329-336. doi:10.1016/j.annemergmed.2007.12.006

[7] Shlipak, M.G., Lyons, W.L., Go, A.S., et al. (1999) Should the electrocardiogram be used to guide therapy for patients with left bundle-branch block and suspected myocardial infarction? Journal of the American Medical Association, 281, 714-719.

[8] Shlipak, M.G., Go, A.S., Frederick, P.D., et al. (2000)
Treatment and outcomes of left bundle-branch block patients with myocardial infarction who present without chest pain. Journal of the American College of Cardiology, 36, 706-712. doi:10.1016/S0735-1097(00)00789-0

[9] Sgarbossa, E.B., et al. (1996) Electrocardiographic diagnosis of evolving acute myocardial infarction in the presence of left bundle-branch block. The New England Journal of Medicine, 334, 481-487.

[10] Sgarbossa, E.B., et al. (1996) Odds Ratio and scores for independent electrocardiographic criteria, for the GUSTO1 investigators. The New England Journal of Medicine, 334, 481-487.

[11] Sgarbossa, E.B., et al. (1996) Flow chart for the predicttion of AMI in the presence of left bundle branch block, with the use of all possible combinations of the three independent electrocardiographic criteria for the GUSTO-1 investigators. The New England Journal of Medicine, 334, 481-487.

[12] Haywood, L. J., et al. (2005) Left bundle branch block in acute myocardial infarction: Benign or malignant? Journal of the American College of Cardiology, 46, 39-41. doi:10.1016/i.jacc.2005.04.002 